\title{
IMA Commission on New Minerals, Nomenclature and Classification (CNMNC)
}

\author{
NEWSLETTER 3
}

\section{New minerals and nomenclature modifications approved in 2010}

P. A. Williams ${ }^{1}$ (Chairman, CNMNC), F. HATERT $^{2}$ (Vice-Chairman, CNMNC), M. PASERo ${ }^{3}$ (Vice-Chairman, CNMNC) AND S. J. Mills ${ }^{4}$ (Secretary, CNMNC)

1 School of Natural Sciences, University of Western Sydney, Locked Bag 1797, Penrith South DC, NSW 1797, Australia - p.williams@uws.edu.au

2 Laboratoire de Minéralogie, Université de Liège, B-4000 Liège, Belgium - fhatert@ulg.ac.be

3 Dipartimento di Scienze della Terra, Università degli Studi di Pisa, Via Santa Maria 53, I-56126 Pisa, Italy pasero@dst.unipi.it

4 Department of Earth and Ocean Sciences, University of British Columbia, Vancouver BC, Canada V6T 1Z4 smills@eos.ubc.ca

The information given here is provided by the IMA Commission on New Minerals, Nomenclature and

Classification for comparative purposes and as a service to mineralogists working on new species.

Each mineral is described in the following format:

Mineral name, if the authors agree on its release prior to the full description appearing in press

Chemical formula

Type locality

Full authorship of proposal

E-mail address of corresponding author

Relationship to other minerals

Crystal system, Space group; Structure determined, yes or no

Unit-cell parameters

Strongest lines in the X-ray powder-diffraction pattern

Type specimen repository and specimen number Citation details for the mineral prior to publication of full description

It is still a requirement for the authors to publish a full description of the new mineral.

NO OTHER INFORMATION WILL BE RELEASED BY THE COMMISSION

\section{Proposals approved in May 2010}

IMA No. 2010-005

$\mathrm{Fe}_{4}\left(\mathrm{SO}_{4}\right) \mathrm{O}_{2}(\mathrm{OH})_{6} \cdot 2 \mathrm{H}_{2} \mathrm{O}$

Cava del Ferro-Trimpello, Fornovolasco, Vergemoli, Apuan Alps, Tuscany, Italy

Cristian Biagioni*, Elena Bonaccorsi and Paolo Orlandi

E-mail: biagioni@dst.unipi.it

New structure type; known synthetic phase

Monoclinic: $C 2 / \mathrm{m}$; structure determined $a=16.085(2), b=3.054(1), c=10.929(2) \AA$, $\beta=93.78(1)^{\circ}$

8.03(s), 4.37(m), 3.989(m), 3.343(mw), 2.633(mw)

Type material is deposited in the Museo di Storia Naturale e del Territorio, University of Pisa, Via Roma 79, Calci (PI), Italy, catalogue number 19300

How to cite: Biagioni, C., Bonaccorsi, E. and Orlandi, P. (2010) IMA 2010-005. CNMNC Newsletter, June 2010, page 577; Mineralogical Magazine, 74, 577-579. 


\section{P. A. WILLIAMS ET AL.}

IMA No. 2010-006

Hermannroseite

$\mathrm{CaCu}\left(\mathrm{PO}_{4}\right)(\mathrm{OH})$

Tsumeb mine, Tsumeb, Namibia

Jochen Schlüter* and Dieter Pohl

*E-mail: Jochen.Schlueter@uni-hamburg.de

Phosphate analogue of conichalcite

Orthorhombic: $P 2{ }_{1} 2_{1} 2_{1}$; structure determined

$a=7.328(7), b=5.769(6), c=9.123(7) \AA$

$5.710(56), 4.057(37), 3.663(21), 3.092(63)$,

2.854(29), 2.808(100), 2.571(73), 2.525(36)

Type material is deposited in the Mineralogical Museum of the University of Hamburg, Hamburg, specimen number TS 637

How to cite: Schlüter, J. and Pohl, D. (2010) Hermannroseite, IMA 2010-006. CNMNC Newsletter, June 2010, page 578; Mineralogical Magazine, 74, 577-579.

\section{IMA No. 2010-008}

Cuprokalininite

$\mathrm{CuCr}_{2} \mathrm{~S}_{4}$

Pereval marble quarry, near Sludyanka, Irkutsk region, Siberia, Russia $\left(51^{\circ} 37^{\prime} \mathrm{N} 103^{\circ} 38^{\prime} \mathrm{E}\right)$

L.Z. Reznitsky, E.V. Sklyarov, Z.F. Ushchapovskaya, L.F. Suvorova, Yu.S. Polekhovsky, P. Dzierżanowski and Igor G. Barash*

*E-mail: garry@crust.irk.ru

Thiospinel

Cubic: $F d \overline{3} m$; known structure type

$a=9.814(2) \AA$

3.44(6), 2.94(10), 2.44(6), 1.884(9), 1.731(10),

1.133(6), 1.098(6), 1.030(6), 1.002(10)

Type material is deposited in the Fersman Mineralogical Museum of the Russian Academy of Sciences, specimen number 3886/1-3

How to cite: Reznitsky, L.Z., Sklyarov, E.V., Ushchapovskaya, Z.F., Suvorova, L.F., Polekhovsky, Yu.S., Dzierżanowski, P. and Barash, I.G. (2010) Cuprokalininite, IMA 2010-008. CNMNC Newsletter, June 2010, page 578; Mineralogical Magazine, 74, $577-579$

\section{IMA No. 2010-009}

Natropharmacoalumite

$\mathrm{NaAl}_{4}\left(\mathrm{AsO}_{4}\right)_{3}(\mathrm{OH})_{4} \cdot 4 \mathrm{H}_{2} \mathrm{O}$

Maria Josefa mine, near Rodalquilar, Andalusia region, Spain $\left(36^{\circ} 51^{\prime} 30 \mathrm{~N} 2^{\circ} 5^{\prime} 2 \mathrm{~W}\right)$

Mike S. Rumsey*, Stuart J. Mills and John Spratt

*E-mail: m.rumsey@nhm.ac.uk

Pharmacosiderite group
Cubic: $P \overline{4} 3 m$; structure determined

$a=7.7280(3) \AA$

$7.759(100), 4.473(40), 3.870(50), 3.459(6)$, 3.158(6), 2.736(6), 2.446(9), 2.331(12)

Type material is deposited in the Natural History Museum in London, specimen number BM 2009,161

How to cite: Rumsey, M.S., Mills, S.J. and Spratt, J. (2010) Natropharmacoalumite, IMA 2010-009. CNMNC Newsletter, June 2010, page 578; Mineralogical Magazine, 74, 577-579.

\section{NOMENCLATURE PROPOSAL APPROVED IN MAY 2010}

Nomenclature of the pyrochlore supergroup minerals

Re-examination and redefinition of pyrochlore end-members and potential new end-members and species has been undertaken, including a classification guide for naming future species.

\section{Proposals approved in June 2010}

IMA No. 2010-007

Greenwoodite

$\left(\mathrm{Ba}, \mathrm{V}^{3+} \mathrm{O}\right)_{2} \mathrm{~V}_{9}^{3+}\left(\mathrm{Fe}^{3+}, \mathrm{Fe}^{2+}\right)_{2} \mathrm{Si}_{2} \mathrm{O}_{22}$

Wigwam deposit, Akolkolex River area British Columbia, Canada (50 $\left.52^{\prime} 48^{\prime \prime} \mathrm{N} 117^{\circ} 58^{\prime} 04^{\prime \prime} \mathrm{W}\right)$

Paul R. Bartholomew*, Franco Mancini, George E. Harlow, Christopher Cahill, Nicholas Deifel and Heinz-Jrgen Bernhardt

*E-mail: pbartholomew@newhaven.edu

New structure type

Trigonal: $P \overline{3} m 1$; structure determined

$a=5.7500(6), c=14.4590(9) \AA$

$2.925(100), 2.875(38), 2.672(23), 2.469(35)$,

2.354(28), 2.212(28), 1.669(26), 1.438(35)

Type material is deposited in the American Museum of Natural History, New York, catalogue number 109839

How to cite: Bartholomew, P.R., Mancini, F., Harlow, G.E., Cahill, C., Deifel, N. and Bernhardt, H.-Z. (2010) Greenwoodite, IMA 2010-007. CNMNC Newsletter, June 2010, page 578; Mineralogical Magazine, 74, 577-579.

\section{IMA No. 2010-010 \\ Naquite}

FeSi

Orebody 31, Luobusa mining district, Qusong County, Tibet $\left(29^{\circ} 5^{\prime} \mathrm{N} 92^{\circ} 5^{\prime} \mathrm{E}\right)$

Shi Ni-cheng*, Li Guo-wu, Bai Wen-ji, Xiong Ming, Yang Jing-su, Fang Qing-son, Ma Zhe-sheng and Rong He 


\section{NEWSLETTER 3}

*E-mail: shinicheng@vip.sina.com

Known structure type

Cubic: $P 2{ }_{1} 3$

$a=4.486(4) \AA$

3.174(43), 2.592(46), 2.249(25), 2.008(100), 1.831(69), 1.353(28), 1.199(38)

Type material is deposited in the Institute of Geology, Chinese Academy of Geological Sciences, Beijing, People's Republic of China, catalogue number 97-8-2

How to cite: Shi, N.-C., Li, G.-W., Bai, W.-J., Xiong, M., Yang, J.-S., Fang, Q.-S., Ma, Z.-S. and Rong, H. (2010) Naquite, IMA 2010-010. CNMNC Newsletter, June 2010, page 579; Mineralogical Magazine, 74, 577-579.

\section{IMA No. 2010-011}

Linzhiite

$\mathrm{FeSi}_{2}$

Orebody 31, Luobusa mining district, Qusong County, Tibet $\left(29^{\circ} 5^{\prime} \mathrm{N} 92^{\circ} 5^{\prime} \mathrm{E}\right)$

Li Guo-wu*, Shi Ni-cheng, Bai Wen-ji, Xiong Ming, Fang Qing-son and Ma Zhe-sheng

*E-mail: liguowu@126.com

Known synthetic compound

Tetragonal: $P 4 / \mathrm{mmm}$; structure determined $a=2.725(3), c=5.202(10) \AA$

$5.150(95), 2.373(66), 1.895(61), 1.848(100)$, 1.776(11), 1.704(13), 1.340(15), 1.086(19)

Type material is deposited in the Institute of Geology, Chinese Academy of Geological Sciences, Beijing, People's Republic of China, catalogue number 97-6

How to cite: Li, G.-W., Shi, N.-C., Bai, W.-J., Xiong, M., Fang, Q.-S. and Ma, Z.-S. (2010) Linzhiite, IMA 2010-011. CNMNC Newsletter, June 2010, page 579; Mineralogical Magazine, 74, 577-579.

\section{IMA No. 2010-012}

\section{Coralloite}

$\mathrm{Mn}^{2+} \mathrm{Mn}_{2}^{3+}\left(\mathrm{AsO}_{4}\right)_{2}(\mathrm{OH})_{2} \cdot 4 \mathrm{H}_{2} \mathrm{O}$

Monte Nero mine, Rocchetta Vara, La Spezia,
Liguria, Italy

Athos Maria Callegari*, Massimo Boiocchi, Marco E. Ciriotti and Corrado Balestra

*E-mail: athosmaria.callegari@unipv.it

Related to arthurite and whitmoreite

Triclinic: $P 1$

$a=5.5828(7), b=9.7660(13), c=5.5455(7) \AA$, $\alpha=94.467(3), \beta=111.348(2), \gamma=93.85(2)^{\circ}$

$9.710(100), 5.166(77), 5.136(80), 3.342(65)$, 3.324(34), 2.873(22), 2.631(23), 2.565(22)

Type material is deposited in the Mineralogical Museum of the University of Pavia, catalogue number 2010/001

How to cite: Callegari, A.M., Boiocchi, M., Ciriotti, M.E. and Balestra, C. (2010) Coralloite, IMA 2010-012. CNMNC Newsletter, June 2010, page 579; Mineralogical Magazine, 74, $577-579$

\section{IMA No. 2010-014}

Hydroniumpharmacosiderite

$\left(\mathrm{H}_{3} \mathrm{O}\right) \mathrm{Fe}_{4}\left(\mathrm{AsO}_{4}\right)_{3}(\mathrm{OH})_{4} \cdot 4 \mathrm{H}_{2} \mathrm{O}$

Cornwall, United Kingdom, probably from a mine in the St Day mines, Wheal Gorland group Stuart J. Mills*, Anthony R. Kampf, Peter A. Williams, Peter Leverett, Glenn Poirier, Mati Raudsepp and Carl A. Francis

*E-mail: smills@eos.ubc.ca

Pharmacosiderite group

Cubic: $P \overline{4} 3 m$; structure determined $a=7.9587(2) \AA$

$8.050(100), 4.628(22), 4.005(14), 3.265(35)$, 2.830(23), 2.528(19), 2.412(30), 1.787(14)

Type material is deposited in the Harvard Mineralogical Museum, catalogue number 142784

How to cite: Mills, S.J., Kampf, A.R., Williams, P.A., Leverett, P., Poirier, G. , Raudsepp, M. and Francis, C.A. (2010) Hydroniumpharmacosiderite, IMA 2010-014. CNMNC Newsletter, June 2010, page 579; Mineralogical Magazine, 74, 577-579. 